\title{
Monotonicity of fuzzy rule bases: On differences between graded and non-graded approaches
}

\author{
Martina Daňková ${ }^{1}$ Martin Štěpnička ${ }^{1}$ Bernard De Baets $^{2}$ \\ ${ }^{1}$ Institute for Research and Applications of Fuzzy Modeling, University of Ostrava, Ostrava, Czech Republic \\ ${ }^{2}$ Department of Applied Mathematics, Biometrics and Process Control, Ghent University, Gent, Belgium
}

\begin{abstract}
This contribution provides an exhaustive explanation of the graded approach to the monotonicity property (of a fuzzy relation) and its non-graded version. Our framework is algebraic. We present a wide variety of examples to support our claims relating to the well defined notion and suitability for fuzzy rule base applications and moreover, to improve the readability of the related mathematical formulae.
\end{abstract}

Keywords: Fuzzy relations, Monotonicity, Functionality, Fuzzy Class Theory

\section{Monotone fuzzy rule bases}

The notion of monotonicity of fuzzy relations has been introduced in [1] and it is closely related to the notion of fuzzy function [2]. More precisely, the logical structure of the definition of the monotonicity is identical with the one given for the functionality and as shown in [1]. And hence, the results known in one field can be transferred to the second if appropriate requirements are met.

Historically, monotonicity was connected with fuzzy rule bases (FRBs) only. Monotone fuzzy rule bases that express monotone dependencies are often found in practical applications e.g. [3, 4]. In the center of attention, there are problems with non-monotone outputs (in a classical sense of nonmonotone function) of a monotone FRB. These problems are closely related to the chosen model of a fuzzy rule base as well as to the chosen defuzzification method (not considered within this contribution). There are various lines of research following particular models of a monotone FRB. For example, Mamdani-Assilian models of monotone FRBs were investigated in $[5,6,7,8]$ where it has been observed that not always they lead to monotone resulting functions. Another line relates to the $\mathrm{im}$ plicative (or alternatively called gradual [9]) models of FRBs, see [10, 4].

Let us consider that a fuzzy rule base $\mathcal{R}$ is simply constituted of a collection of pairs of fuzzy sets $\mathcal{R}=$

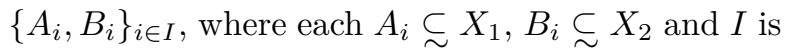
some finite index set. The choice of a model of $\mathcal{R}$ is reflected in the resulting fuzzy relation $R$.

The main idea of the following definition of the monotone FRB is to approximately describe an ideal monotone dependency using a finite number of fuzzy rules.

Definition 1 Let $\leq_{j}$ be a crisp ordering of fuzzy sets on $X_{j}$ for each $j=1,2$. A rule base $\mathcal{R}$ is said to be monotone if for all $i, j \in I$ :

$$
\text { if } A_{i} \leq_{1} A_{j} \text { then } B_{i} \leq_{2} B_{j} .
$$

Remark that in the above definition

- no model of the FRB is assumed;

- no defuzzification is employed to the considered FRB;

- and therefore, no resulting function is taken into account.

It means that this definition reflects only a dependency between "ordered" couples $\left\{A_{i}, A_{j}\right\}$ and $\left\{B_{i}, B_{j}\right\}$.

Moreover, this definition of monotone FRB is non-graded (or crisp). What does it mean?

\section{Graded and non-graded properties}

Traditionally, we are used to deal with properties that are uniquely determined (they are either true or false). Although the great development in the theory of fuzzy sets and fuzzy logic has been made there exist only a few works that enjoy the full beauty of the graded world. It is caused by the following facts:

- First, an appropriate tool for handling higher order objects such as fuzzy sets of fuzzy sets, or expressing graded properties of such complex objects has been proposed recently [11, 12].

- And since there was no simple unified way how to generalize crisp properties of fuzzy sets to fuzzy (graded) ones, a usefulness of the graded approach to such properties has not been generally accepted yet.

Exemplary contributions that provide a systematic study of particular graded properties and show results in the form of graded theorems developed within $\mathrm{FCT}$ are $[13,14,15,2,1]$. A motivation for using graded properties is also explained there. Generally, there was a need of having an opportunity to use some methods that were originally developed for a class of objects that fulfill some crisp 


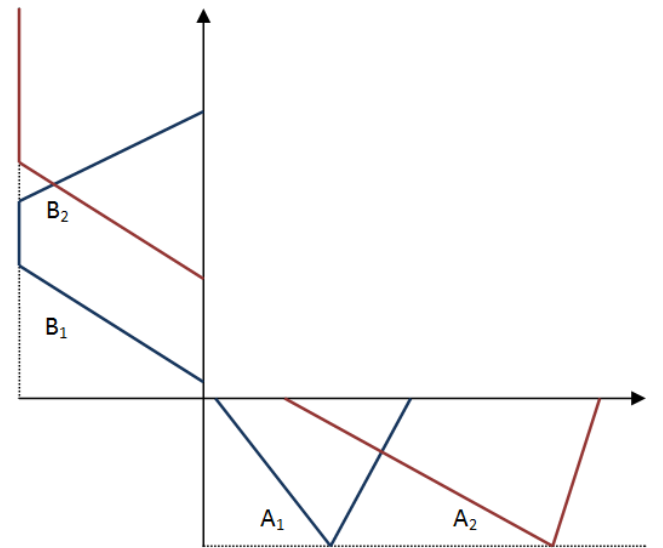

(a) $\mathcal{R}_{1}$ with various types of fuzzy sets

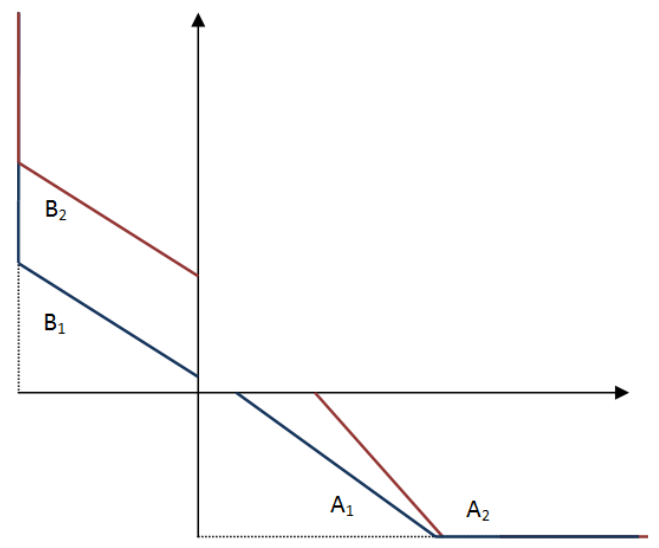

(b) $\mathcal{R}_{2}$ with right-open fuzzy intervals

Figure 1: Examples of monotone FRB.

property (one or more), for a wider class of objects that violates the given crisp property but they satisfy a graded variant of this property in a reasonable high degree.

Let us demonstrate it on Definition 1: we consider a FRB and we examine its monotonicity classically - using classical mathematical logic. We first introduce an appropriate partial order $\leq_{f}$ on fuzzy sets based on the ordering of their $\alpha$-cuts. For a fuzzy set $C$ on a universe $U$, its $\alpha$-cut $[C]_{\alpha}$ is the subset of $U$ defined as

$$
\left.\left.[C]_{\alpha}=\{x \in U \mid C(x) \geq \alpha\}, \text { for any } \alpha \in\right] 0,1\right] .
$$

Definition 2 Let $U$ be a linear vector space over the real numbers $\mathbb{R}$. Given two convex ${ }^{1}$ fuzzy sets $C_{1}$ and $C_{2}$ on a universe $U \subseteq \mathbb{R}$, we say that $C_{1} \leq_{f} C_{2}$ if for any $\alpha \in] 0,1]$ it holds that

$$
\inf \left[C_{1}\right]_{\alpha} \leq \inf \left[C_{2}\right]_{\alpha} \text { and } \sup \left[C_{1}\right]_{\alpha} \leq \sup \left[C_{2}\right]_{\alpha} .
$$

On Figure 1, we see two different examples of monotone FRB in the sense of Definition 1, i.e., where $\leq_{1,2}$ are identical with $\leq_{f}$.

\footnotetext{
${ }^{1}$ A fuzzy set $A \in \mathcal{F}(U)$ is called convex if the inequality$$
A\left(\lambda x_{1}+(1-\lambda) x_{2}\right) \geq A\left(x_{1}\right) \wedge A\left(x_{2}\right)
$$

holds for all $x_{1}, x_{2} \in U$ and all $\lambda \in[0,1]$.
}

For the sequel, assume a residuated lattice

$$
\mathcal{L}=\langle L, \&, \rightarrow, \wedge, \vee, 0,1\rangle,
$$

a structure for truth values with the following table providing a possible reading using a natural language:

\begin{tabular}{|c|c|}
\hline Reading & Connective \\
\hline \hline IF $\ldots$ THEN $\ldots$ & $\ldots \rightarrow \ldots$ \\
$\ldots$ AND $\ldots$ & $\ldots \& \ldots$ \\
$\ldots$ AND $\ldots$ & $\ldots \wedge \ldots$ \\
$\ldots$ OR $\ldots$ & $\ldots \vee \ldots$ \\
\hline
\end{tabular}

In the process of fuzzification of (1), we replace crisp orderings $\leq_{1,2}$ by fuzzy orderings $\preccurlyeq 1_{1,2}$ first. And consequently, the non-graded formalism has to be exchanged by graded one. The result is following:

$$
\left(A_{i} \preccurlyeq{ }_{1} A_{j}\right) \rightarrow\left(B_{i} \preccurlyeq{ }_{2} B_{j}\right),
$$

which can be read as

$$
\text { IF }\left(A_{i} \preccurlyeq{ }_{1} A_{j}\right) \text { THEN }\left(B_{i} \preccurlyeq{ }_{2} B_{j}\right) \text {. }
$$

By (3), we can immediately calculate the degree in which two couples of fuzzy sets preserve the given fuzzy orderings or, in other words, how much two fuzzy rules from FRB $\mathcal{R}$ are monotone. Hence, we call it graded monotonicity for particular couples of fuzzy sets (or fuzzy rules represented by) $\left\{A_{i}, B_{i}\right\}$ and $\left\{A_{j}, B_{j}\right\}$.

Whenever we investigate (absolute) truth of (3) we put it equal to 1 and obtain that it is fulfilled if and only if

$$
\left(A_{i} \preccurlyeq 1 A_{j}\right) \leq\left(B_{i} \preccurlyeq_{2} B_{j}\right)
$$

that is exactly the non-graded monotonicity for the particular couples of fuzzy sets $\left\{A_{i}, B_{i}\right\}$ and $\left\{A_{j}, B_{j}\right\}$. In this case, we have that the non-graded monotonicity do not exhibit degrees and it is either true or false. Moreover, it is a special case of the graded monotonicity.

Inspecting Definition 1, there is still some work to be done, namely, quantification over indexes $i, j$, which is implemented by means of $\wedge$. Consequently, the whole Definition 1 is translated to

$$
\bigwedge_{i, j \in I}\left[\left(A_{i} \preccurlyeq 1 A_{j}\right) \rightarrow\left(B_{i} \preccurlyeq 2 B_{j}\right)\right],
$$

denoted by MonRB $\preccurlyeq_{1,2}(\mathcal{R})$. That is called graded monotonicity of a FRB $\mathcal{R}$, where evaluation of (5) gives a degree of this property. Using the following language:

\begin{tabular}{|c|c|}
\hline Reading & Operation \\
\hline \hline$A$ is smaller than $A^{\prime}$ & $A \preccurlyeq{ }_{1} A^{\prime}$ \\
$B$ is smaller than $B^{\prime}$ & $B \preccurlyeq{ }_{2} B^{\prime}$ \\
FOR ALL $i \ldots$ & $\wedge_{i} \ldots$ \\
\hline
\end{tabular}


we can read (5) as

\section{"FOR ALL $i, j \in I$}

[IF $\left(A_{i}\right.$ is smaller than $\left.A_{j}\right)$ THEN

$$
\left(B_{i} \text { is smaller than } B_{j}\right) \text { " }
$$

Finally, let us provide a formal definition of graded monotonicity that directly involves degrees.

Definition 3 Let $\mathcal{L}$ be a residuated lattice of the form (2), $\preccurlyeq_{1(2)}$ be a binary fuzzy relation over a non-empty set $X_{1(2)}$ and $\mathcal{R}$ be a FRB. If

$$
a=\bigwedge_{i, j \in I}\left[\left(A_{i} \preccurlyeq{ }_{1} A_{j}\right) \rightarrow\left(B_{i} \preccurlyeq_{2} B_{j}\right)\right],
$$

then we say that $\mathcal{R}$ is a-monotone w.r.t. $\preccurlyeq 1,2$.

Let us comment on the above definition:

- Assuming a class of FRBs, e.g. $\left\{\mathcal{R}_{k}\right\}_{k}$, we distinguish a fuzzy sub-class of monotone FRBs, where we assign to each FRB $\mathcal{R}_{k}$ a particular degree $a_{k}$ by means of (6), i.e., $a_{k}=$ $\operatorname{MonRB}_{\preccurlyeq 1,2}\left(\mathcal{R}_{k}\right)$.

- The degree of monotonicity can be evaluated for an arbitrary $\mathcal{R}$.

- If $a=1$ for $\mathcal{R}$, we can say $\mathcal{R}$ is monotone and if $a=0$, we can safely say $\mathcal{R}$ is not monotone.

- The definition deals with degrees. We may proceed directly without specifying degrees just by processing the syntax of a specific fuzzy logic. This way has been elaborated in [1].

- The crisp $\leq_{1,2}$ are also allowed as a special case of fuzzy relations $\preccurlyeq r_{1,2}$. Then $a \in\{0,1\}$ and evaluation of this property produces the same result as checking the classical monotonicity of $\mathcal{R}$ given by Definition 1 . It is $a=1$ for monotone $\mathcal{R}$ in the sense of Definition 1 and $a=0$ for non-monotone $\mathcal{R}$.

Now, let us investigate the absolute truth of (6):

$$
\bigwedge_{i, j \in I}\left(A_{i} \preccurlyeq 1 A_{j}\right) \rightarrow\left(B_{i} \preccurlyeq 2 B_{j}\right)=1,
$$

it follows that none of the implications can be smaller than 1

$$
\left(A_{i} \preccurlyeq_{1} A_{j}\right) \rightarrow\left(B_{i} \preccurlyeq_{2} B_{j}\right)=1,
$$

for all $i, j \in I$, and equivalently

$$
\left(A_{i} \preccurlyeq 1 A_{j}\right) \leq\left(B_{i} \preccurlyeq_{2} B_{j}\right),
$$

for all $i, j \in I$, which are the principal inequalities for the definition of the non-graded monotonicity of $\mathcal{R}$. Let us summarize it in the following definition:

Definition 4 Let $\mathcal{L}, \preccurlyeq 1(2)$ and $\mathcal{R}$ be as in Definition 3. If for each $i, j \in I$

$$
\left(A_{i} \preccurlyeq{ }_{1} A_{j}\right) \leq\left(B_{i} \preccurlyeq_{2} B_{j}\right)
$$

is valid then we say that $\mathcal{R}$ fulfills non-graded monotonicity (or it is non-graded monotone) w.r.t. $\preccurlyeq 1,2$.
Example 5 Let us consider an example of a fuzzy rule base $\mathcal{R}$ consisting of two fuzzy rules $\left\{A_{i}, B_{i}\right\}, i=1,2$, that is monotone in the sense of (8). First of all, we have to define fuzzy orderings $\preccurlyeq 1,2$ of fuzzy sets. For this, we adopt the definition introduced by $U$. Bodenhofer et al. (see [16, 17]) that defines the fuzzy order $\preccurlyeq: \mathcal{F}^{2}(U) \rightarrow[0,1]$ of two fuzzy sets $C, D \in \mathcal{F}(U)$ as follows:

$$
\begin{array}{r}
C \preccurlyeq D=\bigwedge_{u \in U}[(\operatorname{ATL}(D)(u) \rightarrow \operatorname{ATL}(C)(u)) \& \\
(\operatorname{ATM}(C)(u) \rightarrow \operatorname{ATM}(D)(u))]
\end{array}
$$

where ATL, ATM denote at-least and at-most modifiers [18], respectively, that are given as follows:

$$
\begin{aligned}
\operatorname{ATL}(C) & =\bigvee(C(t) \& t \preceq u), \\
\operatorname{ATM}(C) & =\bigvee(C(t) \& u \preceq t)
\end{aligned}
$$

for arbitrary $C \in \mathcal{F}(U)$. The symbol $\preceq$ generally denotes a $T-E-$ ordering, i.e., a fuzzy ordering with respect to a $t$-norm $T$ and $E$ is a fuzzy equivalence relation.

For this example, it is sufficient to consider $\preceq$ to be equivalent to the ordering of real numbers $\leq$ and hence:

$$
\begin{aligned}
& \operatorname{ATL}(C)=\bigvee_{t \leq u} C(t), \\
& \operatorname{ATM}(C)=\bigvee_{u \leq t} C(t) .
\end{aligned}
$$

Further, let the standard Lukasiewicz algebra $\mathcal{L}$ be the underlying structure for truth values, $X_{1}=$ $X_{2}=[0,4]$ and the involved fuzzy sets be given as follows:

$$
\begin{aligned}
& A_{1}= \begin{cases}(x-1) \vee 0, & x \in[1,2], \\
(3-x) \vee 0, & x \in[2,3],\end{cases} \\
& A_{2}= \begin{cases}(x-2) \vee 0, & x \in[2,3], \\
(4-x) \vee 0, & x \in[3,4],\end{cases} \\
& B_{1}= \begin{cases}(y-1) \vee 0, & y \in[1,2], \\
(3-y) \vee 0, & y \in[2,3],\end{cases} \\
& B_{2}= \begin{cases}(y-2) \vee 0, & y \in[2,3], \\
(4-y) \vee 0, & y \in[3,4] .\end{cases}
\end{aligned}
$$

It is easy to show that $A_{1} \preccurlyeq A_{2}=1, A_{2} \preccurlyeq A_{1}=0$ and that also $B_{1} \preccurlyeq B_{2}=1, B_{2} \preccurlyeq B_{1}=0$ and hence, that

$$
\begin{aligned}
& \left(A_{1} \preccurlyeq A_{2}\right) \rightarrow\left(B_{1} \rightarrow B_{2}\right)=1, \\
& \left(A_{2} \preccurlyeq A_{1}\right) \rightarrow\left(B_{2} \rightarrow B_{1}\right)=1
\end{aligned}
$$




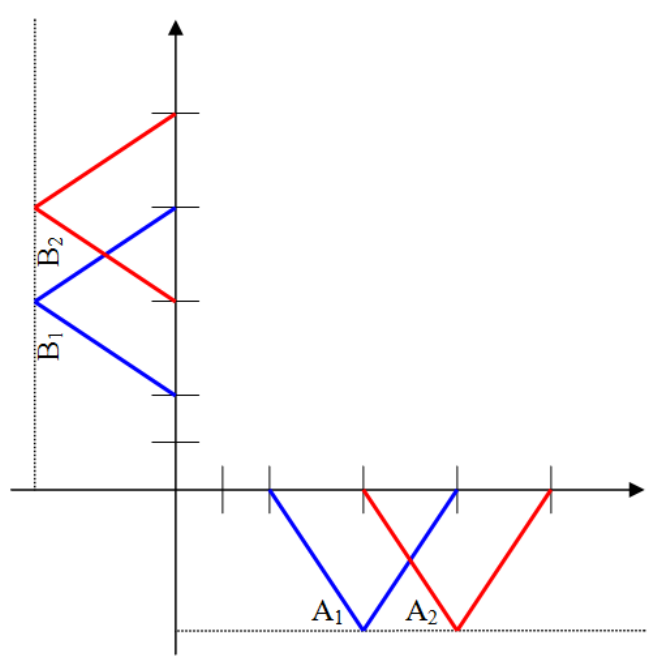

Figure 2: Non-graded monotone fuzzy rule base $\mathcal{R}$ from Example 5.

which gives

$$
\bigwedge_{i, j}\left[\left(A_{i} \preccurlyeq A_{j}\right) \rightarrow\left(B_{i} \preccurlyeq B_{j}\right)\right]=1 .
$$

So, fuzzy rule base $\mathcal{R}$ is an example of a fuzzy rule base that is non-graded monotone w.r.t. $\preccurlyeq 1,2$, i.e., it fully obeys (8).

Let us note that the fuzzy rule base in Example 5 was chosen to meet also the original definition of a monotone fuzzy rule base, i.e., to meet Definition 1. But the given fuzzy rule base may be easily modified on order to lose this property but still in such a way that it is monotone (non-graded monotone, to be precise) according to the newly proposed Definition 4.

Example 6 Let us consider a fuzzy rule base $\mathcal{R}^{\prime}$ consisting of two fuzzy rules $\left\{A_{i}, B_{i}\right\}, i=1,2$, that is monotone in the sense of (8) and it is not monotone in the sense of Definition 1. Let us keep all the setting (algebra, fuzzy ordering etc.) from Examples 5.

Then, let the involved fuzzy sets are slightly modified as follows:

$$
\begin{aligned}
& A_{1}= \begin{cases}(x-1) \vee 0, & x \in[1,2], \\
(3-x) \vee 0, & x \in[2,3],\end{cases} \\
& A_{2}= \begin{cases}\left(\frac{x-0.5}{2.5}\right) \vee 0, & x \in[0.5,3], \\
(4-x) \vee 0, & x \in[3,4],\end{cases} \\
& B_{1}= \begin{cases}(y-1) \vee 0, & y \in[1,2], \\
(3-y) \vee 0, & y \in[2,3],\end{cases} \\
& B_{2}= \begin{cases}\left(\frac{y-0.5}{2.5}\right) \vee 0, & y \in[0.5,3], \\
(4-y) \vee 0, & y \in[3,4] .\end{cases}
\end{aligned}
$$

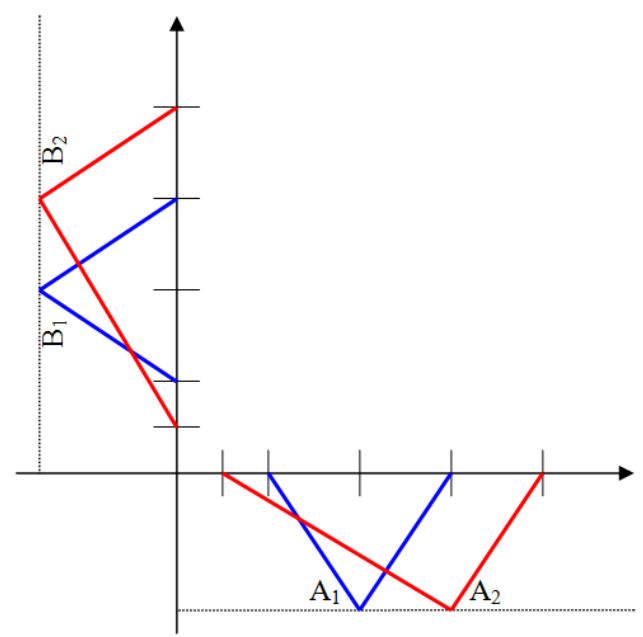

Figure 3: Non-graded monotone fuzzy rule base $\mathcal{R}^{\prime}$ from Example 6.

Then it can be shown that

$$
\begin{aligned}
& \bigwedge_{x \in X_{1}}\left(\operatorname{ATL}\left(A_{2}\right) \rightarrow \operatorname{ATL}\left(A_{1}\right)\right)=0.2 \rightarrow 0=0.8, \\
& \bigwedge_{x \in X_{1}}\left(\operatorname{ATM}\left(A_{1}\right) \rightarrow \operatorname{ATM}\left(A_{2}\right)\right)=1, \\
& \bigwedge_{y \in X_{2}}\left(\operatorname{ATL}\left(B_{2}\right) \rightarrow \operatorname{ATL}\left(B_{1}\right)\right)=0.2 \rightarrow 0=0.8, \\
& \bigwedge_{y \in X_{2}}\left(\operatorname{ATM}\left(B_{1}\right) \rightarrow \operatorname{ATM}\left(B_{2}\right)\right)=1,
\end{aligned}
$$

and hence that both implications hold:

$$
\begin{aligned}
& \left(A_{1} \preccurlyeq A_{2}\right) \rightarrow\left(B_{1} \rightarrow B_{2}\right)=0.8 \rightarrow 1=1, \\
& \left(A_{2} \preccurlyeq A_{1}\right) \rightarrow\left(B_{2} \rightarrow B_{1}\right)=0.8 \rightarrow 1=1
\end{aligned}
$$

which gives

$$
\bigwedge_{i, j}\left[\left(A_{i} \preccurlyeq A_{j}\right) \rightarrow\left(B_{i} \preccurlyeq B_{j}\right)\right]=1 .
$$

So, fuzzy rule base $\mathcal{R}^{\prime}$ is an example of a fuzzy rule base that is non-graded monotone w.r.t. $\preccurlyeq 1,2$, although it could not be called monotone if only the original (crisp) Definition 1 was at disposal.

For the completeness of the information the reader is provided with, let us add an example of a FRB that is not non-graded monotone but that is at least $a$-monotone to some degree $a$.

Example 7 Let us consider an example of a fuzzy rule base $\mathcal{R}^{\prime \prime}$ consisting of two fuzzy rules $\left\{A_{i}, B_{i}\right\}, i=1,2$. Let us keep all the setting (algebra, fuzzy ordering etc.) from Examples 5. 


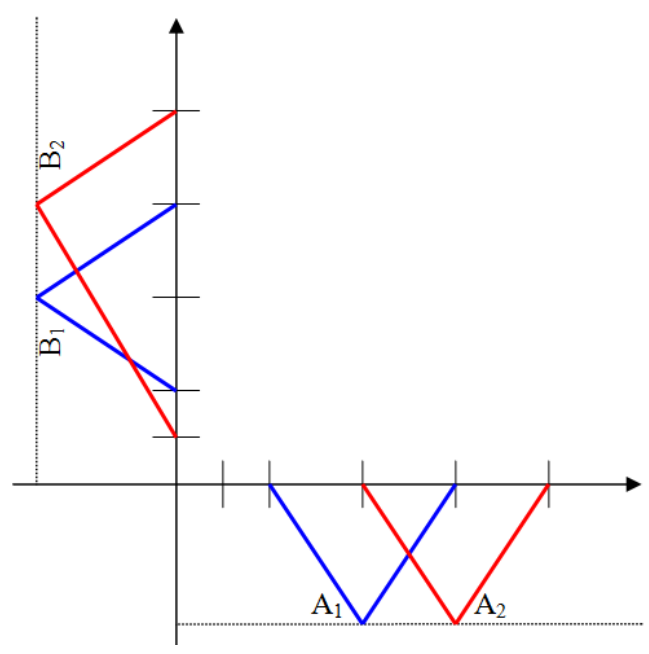

Figure 4: Fuzzy rule base $\mathcal{R}^{\prime \prime}$ from Example 7 that is 0.8 -monotone.

Let the involved fuzzy sets be given as follows:

$$
\begin{aligned}
& A_{1}= \begin{cases}(x-1) \vee 0, & x \in[1,2], \\
(3-x) \vee 0, & x \in[2,3],\end{cases} \\
& A_{2}= \begin{cases}(x-2) \vee 0, & x \in[2,3], \\
(4-x) \vee 0, & x \in[3,4],\end{cases} \\
& B_{1}= \begin{cases}(y-1) \vee 0, & y \in[1,2], \\
(3-y) \vee 0, & y \in[2,3],\end{cases} \\
& B_{2}= \begin{cases}\left(\frac{y-0.5}{2.5}\right) \vee 0, & y \in[0.5,3], \\
(4-y) \vee 0, & y \in[3,4] .\end{cases}
\end{aligned}
$$

It is easy to show that $A_{1} \preccurlyeq A_{2}=1, A_{2} \preccurlyeq A_{1}=$ $0, B_{2} \preccurlyeq B_{1}=0$ and that

$$
\bigwedge_{y \in X_{2}}\left(\operatorname{ATL}\left(B_{2}\right) \rightarrow \operatorname{ATL}\left(B_{1}\right)\right)=0.2 \rightarrow 0=0.8 .
$$

Hence

$$
\begin{aligned}
& \left(A_{1} \preccurlyeq A_{2}\right) \rightarrow\left(B_{1} \rightarrow B_{2}\right)=0.8 \quad(*) \\
& \left(A_{2} \preccurlyeq A_{1}\right) \rightarrow\left(B_{2} \rightarrow B_{1}\right)=1
\end{aligned}
$$

which gives

$$
\bigwedge_{i, j}\left[\left(A_{i} \preccurlyeq A_{j}\right) \rightarrow\left(B_{i} \preccurlyeq B_{j}\right)\right]=0.8 .
$$

So, fuzzy rule base $\mathcal{R}^{\prime \prime}$ is an example of a fuzzy rule base that is not non-graded monotone w.r.t. $\preccurlyeq 1,2$, i.e., (8) fails by $\left(^{*}\right)$. On the other, hand, it is monotone at least to some degree, particularly, to the degree 0.8. Hence, $\mathcal{R}^{\prime \prime}$ is 0.8-monotone.

\subsection{Monotone fuzzy rule bases and their relationship to monotone functions}

For the sequel, assume a crisp function $f$ from the set of all fuzzy sets on $X_{1}$ denoted by $\mathcal{F}\left(X_{1}\right)$ into the set of all fuzzy sets on $X_{2}$ denoted by $\mathcal{F}\left(X_{2}\right)$, i.e., $f: \mathcal{F}\left(X_{1}\right) \mapsto \mathcal{F}\left(X_{2}\right)$. Furthermore, let $\approx_{1(2)}$ be a similarity on $\mathcal{F}\left(X_{1(2)}\right), \preccurlyeq 1,2$ be fuzzy orderings of fuzzy sets.

Specially, a FRB can viewed as a collection of information about some function. Let us emphasis that in our case this function is from $\mathcal{F}\left(X_{1}\right)$ into $\mathcal{F}\left(X_{2}\right)$. Taking into account Definition 3 , we can immediately generalize it to the case of graded monotonicity of such function.

Definition 8 A crisp function $f: \mathcal{F}\left(X_{1}\right) \mapsto$ $\mathcal{F}\left(X_{2}\right)$ is said to be a-monotone w.r.t. $\preccurlyeq 1,2$ if

$$
a=\bigwedge_{X, X^{\prime} \in \mathcal{F}\left(X_{1}\right)}\left[\left(X \preccurlyeq 1 X^{\prime}\right) \rightarrow\right.
$$

$$
\left.\left(f(X) \preccurlyeq 2 f\left(X^{\prime}\right)\right)\right] .
$$

We will shortly denote the right side of the above equation by $\mathrm{Mon}_{\preccurlyeq 1,2}(f)$.

This definition perfectly translates the classical definition of a monotone function to the graded form. Mon $_{\preccurlyeq 1,2}(f)$ can be translated as

\section{("For AlL $\left.X, X^{\prime}\right)\left[\operatorname{IF}\left(X\right.\right.$ IS SMALleR Than $\left.X^{\prime}\right)$} THEN $f(X)$ IS SMALLER THAN $\left.f\left(X^{\prime}\right)^{\prime \prime}\right]$

Next, we can define the following binary fuzzy relation on $\mathcal{F}\left(X_{1}\right) \times \mathcal{F}\left(X_{2}\right)$ :

$$
R_{f}(X, Y)=Y \approx_{2} f(X),
$$

which is closely related to the notion of the fuzzy function. The known results (see e.g. [19] or [20]) related to that notion can be transferred to higher order objects (such as fuzzy sets of fuzzy sets) and hence, we have that $R_{f}$ is

- extensional:

$$
\left(X \approx_{1} X^{\prime}\right) \&\left(Y \approx_{2} Y^{\prime}\right) \& R_{f}(X, Y) \leq R_{f}\left(X^{\prime}, Y^{\prime}\right)
$$

is valid for an arbitrary $X, X^{\prime}, Y, Y^{\prime}$ from the respective domains;

- and functional:

$$
\left(X \approx_{1} X^{\prime}\right) \& R_{f}(X, Y) \& R_{f}\left(X^{\prime}, Y^{\prime}\right) \leq\left(Y \approx_{2} Y^{\prime}\right)
$$

is valid for an arbitrary $X, X^{\prime}, Y, Y^{\prime}$ from the respective domains.

Let us consider a fuzzy rule base

$$
\mathcal{R}_{f}=\left\{A_{i}, B_{i}\right\}_{i \in I}
$$

consisting of the perfect samples of $f$. It means that for each $i \in I: B_{i} \approx f\left(A_{i}\right)=1$. Then it can be proved an interesting inequality (see [1])

$$
\operatorname{Mon}_{\preccurlyeq 1,2}(f) \leq \operatorname{MonRB}_{\preccurlyeq 1,2}\left(\mathcal{R}_{f}\right) .
$$

It means that 
- the degree of monotonicity of $f$ is smaller or equal than the degree of monotonicity of the rule base based on $f$;

- or informally speaking, the monotonicity of $f$ implies the monotonicity of $\mathcal{R}_{f}$;

- but this not the other way round.

\section{Conclusion}

In this contribution, we have "fuzzified" the monotonicity property of a FRB into the graded one, i.e., $a$-monotonicity where $a$ represents the particular degree in which a given FRB is monotone. Furthermore, it has been extended to an arbitrary function from fuzzy sets to fuzzy sets. Contrary to the previous work [1] where we worked within a logical framework without a direct interpretation using the degrees of truth, we emphasize here an immediate evaluation of grades. This approach may become useful for readers that are not familiar with a formal logical notational style. And moreover, we have presented examples supporting our way of generalization. In this way, we point out that degrees play their role also in this particular problem relating to FRBs and it is not only an artificial generalization. Finally, the last section showed a connection with functions (with a special domain and range) and foreshadowed the promising area for future work.

\section{Acknowledgment}

We gratefully acknowledge support of the projects MSM6198898701 of the MŠMT ČR and IAA108270902 of the GAAV ČR.

\section{References}

[1] M. Štěpnička M. Daňková and B. De Baets. Grades of monotonicity of fuzzy relations and their application to fuzzy rule bases. In 2011 IEEE Symposium Series on Computational Intelligence Proceedings, pages 37-44, Paris, France, April 2011.

[2] M. Daňková. Representation theorem for fuzzy functions - graded form. In Proc. INTERNATIONAL CONFERENCE ON FUZZY COMPUTATION 2010, pages 56-64, Valencia, Spain, October 2010.

[3] E. Van Broekhoven. Monotonicity aspects of linguistic fuzzy models. PhD thesis, Ghent University, Ghent, Belgium.

[4] M. Štěpnička and B. De Baets. Implicationbased models of monotone fuzzy rule bases. Fuzzy Sets and Systems, submitted.

[5] E. Van Broekhoven and B. De Baets. A linguistic fuzzy model with a monotone rule base is not always monotone. In Proc. EUSFLAT$L F A^{\prime} 05$, pages 530-535, Barcelona, Spain, 2005.

[6] E. Van Broekhoven and B. De Baets. On the monotonicity of mamdani-assilian models with two input variables. In NAFIPS'06, Montreal, Canada, pages 102-107, 2006.

[7] E. Van Broekhoven and B. De Baets. Only smooth rule bases can generate monotone mamdani-assilian models under center-ofgravity defuzzification. IEEE Transactions on Fuzzy Systems, 17:1157-1174, 2009.

[8] E. Van Broekhoven and B. De Baets. Monotone mamdani-assilian models under mean of maxima defuzzification. Fuzzy Sets and Systems, 159:2819-2844, 2008.

[9] D. Dubois and H. Prade. What are fuzzy rules and how to use them. Fuzzy Sets and Systems, 84:169-185, 1996.

[10] M. Štěpnička and B. De Baets. Monotonicity of implicative fuzzy models. In Proc. WCCI (FUZZ-IEEE) 2010, pages 2334-2340, Barcelona, Spain, 2010.

[11] Vilém Novák. On fuzzy type theory. Fuzzy Sets and Systems, 149(2):235-273, 2004.

[12] Libor Běhounek and Petr Cintula. Fuzzy class theory. Fuzzy Sets and Systems, 154(1):34-55, 2005.

[13] Libor Behounek, Ulrich Bodenhofer, and Petr Cintula. Relations in fuzzy class theory:: Initial steps. Fuzzy Sets and Systems, 159(14):17291772, 2008. Formal Methods for Fuzzy Mathematics, Approximation and Reasoning, Part I.

[14] Libor Behounek and Martina Dankov? Relational compositions in fuzzy class theory. Fuzzy Sets and Systems, 160(8):1005 - 1036, 2009. Featured Issue: Formal Methods for Fuzzy Mathematics, Approximation and Reasoning, Part II.

[15] Martina Daňková. On approximate reasoning with graded rules. Fuzzy Sets and Systems, 158:652-673, 2007.

[16] U. Bodenhofer, M. De Cock, and E. E. Kerre. Openings and closures of fuzzy preorderings: theoretical basics and applications to fuzzy rule-based systems. International Journal of General Systems, 32(4):343-360, 2003.

[17] U. Bodenhofer. Orderings of fuzzy sets based on fuzzy orderings part ii: generalizations. Mathware \&f soft computing, 15:219-249, 2008.

[18] U. Bodenhofer. Orderings of fuzzy sets based on fuzzy orderings part i: the basic approach. Mathware \& soft computing, 15:201-218, 2008.

[19] Radim Bělohlávek. Fuzzy Relational Systems: Foundations and Principles, volume 20 of IFSR International Series on Systems Science and Engineering. Kluwer Academic/Plenum Press, New York, 2002.

[20] Mustafa Demirci. Fuzzy functions and their fundamental properties. Fuzzy Sets and Systems, 106:239-246, 1999. 